\title{
Ringer'S Lactate Versus Sterofundin $®$ Iso in Paediatric Surgical Patients: The Acid Base and Electrolytes Assessment
}

\author{
Hasyizan Mohamad Hassan ${ }^{1, ~ *, ~ N u r u l ~ A s h i k i n ~ H a s b u l l a h ~}{ }^{2}$, Saedah Ali ${ }^{1}$, Ruwaida Isa ${ }^{2}$ \\ ${ }^{1}$ Department of Anesthesia and Intensive Care, School of Medical Sciences, Health Campus, Universiti Sains Malaysia, Kubang Kerian, \\ Kelantan, Malaysia \\ ${ }^{2}$ Department of Anesthesia and Intensive Care, Hospital Raja Perempuan Zainab 2, Kota Bharu, Malaysia
}

Email address:

miezan82@yahoo.com (H. M. Hassan),hasyizan@usm.my (H. M. Hassan)

${ }^{*}$ Corresponding author

\section{To cite this article:}

Hasyizan M. Hassan, Asyikin N. Hasbullah, Saedah Ali, Ruwaida Isa. Ringer'S Lactate Versus Sterofundin ${ }^{\circledR}$ Iso in Paediatric Surgical Patients: The Acid Base and Electrolytes Assessment. Journal of Anesthesiology. Vol. 6, No. 1, 2018, pp. 33-39. doi: 10.11648/j.ja.20180601.16

Received: January 14, 2018; Accepted: May 14, 2018; Published: July 4, 2018

\begin{abstract}
The ideal intraoperative intravenous fluid in paediatric anaesthesia and surgery is still debatable. This is essential to overcome physiological changes to maintain safety and wellbeing of the patient. A new isotonic "balanced solution" (Sterofundin ${ }^{\circledR}$ ISO) has been introduced to replace existing intraoperative fluid of choice (Ringer's Lactate). The study aimed to observe any changes in electrolytes and acid base after infusion of each study solutions and to evaluate any post infusion electrolytes and acid base differences between both study solutions. The double blinded randomized control trial involved 141 paediatric patients who were subjected to minor surgical procedure of less than 3 hours were randomized into Ringer's Lactate or Sterofundin ${ }^{\circledR}$ ISO group. The electrolytes and acid base parameters were assessed at pre-infusion and post-infusion time. There were significant difference in glucose, potassium $\left(\mathrm{K}^{+}\right)$and chloride $\left(\mathrm{Cl}^{-}\right)$level between pre-infusion and post-infusion values with those given Ringer's Lactate. There were also significant different in $\mathrm{pH}$, base excess, glucose, Calcium $\left(\mathrm{Ca}^{2+}\right)$, Chloride $(\mathrm{Cl})$ and lactate level between pre-infusion and post-infusion values with Sterofundin $\mathbb{}(\mathrm{ISO}$. When comparing the mean difference (pre-infusion and post-infusion) between Ringer's Lactate and Sterofundin ${ }^{\circledR}$ ISO, calcium $\left(\mathrm{Ca}^{2+}\right)$ is the only electrtolyte which found to be significant $(P=0.015)$ with the values of (Means $\pm \mathrm{SD} 0.005 \pm 0.07) \mathrm{mmol} / \mathrm{L}$ and (Means $\pm \mathrm{SD}$ $0.02 \pm 0.06) \mathrm{mmol} / \mathrm{L}$ respectively. Both study solutions showed variable effects of electrolytes and acid base parameters and no fluid was found to be superior in paediatric surgical patients underwent minor surgery of less than three hours.
\end{abstract}

Keywords: Sterofundin ${ }^{\circledR}$ ISO, Ringer’s Lactate, Paediatric, Minor Surgery, Electrolytes, Acid-Base Balance

\section{Introduction}

Fluid therapy in the paediatric surgical patient is designed to provide for different fluid requirement: i.e; deficit, maintenance and ongoing loss in order to achieve adequate tissue perfusion. Over the decades, maintenance of fluid therapy in children has been based on Holiday and Segar protocol [1]. This recommendation is widely accepted and practiced in paediatric populations [2]. However, types of fluid therapy intraoperatively are still controversial and remain debatable. One group favors usage of isotonic fluid while the others defended hypotonic fluid therapy [3].
Although the current trend prefers usage of isotonic solution, most anesthetist still prescribes hypotonic solutions [4]. It is a matter of fact that the risk of hypoglycaemia and fluctuation of the sodium level are the most common issues addressed when dealing with type of fluid therapy in children [5]. Nevertheless, proper consensus should be obtained to prevent iatrogenic hyponatremia among hospitalized paediatric patients $[2,6,7]$.

Ringer's Lactate is slightly hypotonic crystalloid as compared to human plasma. It has the osmolarity of $273 \mathrm{mOsm} / \mathrm{L}$ and further reduced to only $255 \mathrm{mOsm} / \mathrm{L}$ due to incomplete ionization of the lactate salt. Thus, it is used with 
precaution in patients who are at risk of developing cerebral edema [8]. Ringer's Lactate might as well worsening the pulmonary edema in susceptible patients [9]. In addition, it has inadequate concentration of sodium $(130 \mathrm{mmol} / \mathrm{L})$ and slightly higher level of chloride $(112 \mathrm{mmol} / \mathrm{L})$ as compared to the plasma. However, Kamp-Jansen et. al.[10] found no significant changes of sodium level after infusion of Ringer's Lactate in the healthy individual. Apart from that, addition of the lactate which mainly served as a buffer caused doubling of the plasma glucose level in diabetic patients [11]. De Jonghe et al. [12] reported increase lactate level is also major predictor of mortality in patients with liver failure.

In contrast, Sterofundin $\AA$ is a balanced crystalloid which is isoosmolar $(288 \mathrm{mOsm} / \mathrm{L})$ to human plasma. In comparison to other fluids solution, it has the best electrolyte parameters mimicking to human extracellular fluid constituents. However, the concentration of the chloride ion is slightly more in order to reach the physiological osmolarity. Balanced solution was found to cause more stable acid base paramenters and preventing the hyperchloremic metabolic acidosis [13]. Gan et al. [14] found that balanced solution provides a better haemostasis. In the other study, balanced solution was also found to improve gastric perfusion and preservation of renal function $[15,16]$. Boldt et al. $[17,18]$ also demonstrated that the coagulation profiles and the Base Excess status was significantly better in the patients infused with balanced solution. Circulating blood volume and the organ perfusion was found to be superior in patients given with balanced isotonic crystalloid [19]. Furthermore, Sterofundin $(\circledR$ also contains acetate and malate as buffer which is independent on the metabolism by the liver and kidney.

This study was conducted to observe any changes in electrolytes and acid base after infusion of each study solutions and to evaluate any post infusion electrolytes and acid base differences between both study solutions.

\section{Material and Methods}

\subsection{Patients}

Following approval by the ethical committee board (USMKK/PPP/JEPeM/241.3(8) and written consent from the next of kin in accordance to the Declaration of Helsinki, 145 paediatric patients underwent elective short surgical procedure of less than 3 hours were randomized. The sample size was determined (Power \& Sample Size Calculations, Version 3.0.10, Dupont and Plummer, 1997) with $80 \%$ power, $\alpha=0.05, \sigma=4, \Delta=2[20]$ and $10 \%$ dropped out with a total sample of 63 subjects in each group. We included the paediatric patient with the age of 2 to 12 years old who was ASA1 and 2 (American Society of Anaesthesiologist classification) and not on intravenous drip. Major surgery with expected major fluid loss and blood transfusion, patient with history of gastroenteritis at least one week before surgery, patient with colostomy and those patients with significant renal dysfunction were excluded from the study.
Double blinded randomization using the envelope technique was performed before induction of anesthesia in selected patients. The patients were grouped into group A and B:

Group A: Ringer's Lactate, B. Braun, Germany

Group B: Sterofundin®ISO, B. Braun, Germany

The patients were subsequently induced with either intravenous induction or inhalational agent and $20 \mathrm{G}$ to $22 \mathrm{G}$ branula were inserted at the dorsum of the hand. $1.5 \mathrm{cc}$ of venous blood were drawn and analyzed on two occasions i.e; after induction of anaesthesia and before extubation of the patients. The patients were intubated with endotracheal tube (ETT) or Laryngeal Mask Airway (LMA Classic ${ }^{\circledR}$ ). The maintenance of anesthesia was achieved with Isoflurane with the Minimum Alveolar Concentration (MAC) of 1 to 1.2 and mixture of oxygen and air of $30 \%$ and $70 \%$ respectively. The ventilation of the patients were delivered either with positive pressure ventilation (PPV) with Pressure Control mode to achieve tidal volume of approximately $8 \mathrm{mls} / \mathrm{kg}$ or spontaneous ventilation mode.

The maintenance, deficit and ongoing loss fluid therapy were according to the protocol by Holliday M. and Segar W. [1] The study solutions were administered during the maintenance phase of anaesthesia. The patients' temperatures were kept at least at $36 \mathrm{C}$ with active warming methods. No other solutions were infused except the study solution. Capillary blood glucose was monitored at hourly interval and dextrose containing solution was administered if the blood sugar falls below $3 \mathrm{mmol} / \mathrm{L}$.

Once the surgeries were completed, the study solutions were discontinued and the second blood samples were obtained at the hand or antecubital fossa contralateral to the site of fluid infusion. The patients were subsequently reversed with standard reversal; IV atropine $0.02 \mathrm{mg} / \mathrm{kg}$ and neostigmine $0.04 \mathrm{mg} / \mathrm{kg}$ if the non-depolarizing muscle relaxants were previously used. Finally, the patients were discharged to the ward after 30 minutes of observation in recovery bay.

\subsection{Measurement}

The venous blood samples were taken before induction and reversal of anaesthesia. The Renal Function Tests (RFT) were taken and the concentrations of serum $\mathrm{Na}+, \mathrm{Cl}-, \mathrm{K}+$ and $\mathrm{Ca} 2+$ were measured using the same blood samples (COBAS Integra ${ }^{\circledR} 800$ system, Roche, USA. These samples were also analyzed for $\mathrm{pH}, \mathrm{PaCO} 2, \mathrm{HCO} 3-$, standard base excess (SBE) and serum lactate (Lac), all integrated in the blood gas analyzer (GEM Premier 3000, Instrumentation Laboratory, USA).

\subsection{Statistics}

Statistical analysis was carried out with SPSS version 12.0. The results were opresented in mean and standard deviation. The demographic data in between groups were compared with independent $\mathrm{t}$ test or $\kappa 2$ test where appropriate. The intragroup and intergroup differences were analyzed with paired t-test, independent t-test and Pearson Correlation. The 
results were expressed as mean \pm standard deviation or frequencies as appropriate. The level of significance was determined as $\mathrm{p}<0.05$.

\section{Results}

We recruited a total of 145 paediatric patients who underwent uncomplicated elective minor surgical procedure of less than three hours duration. However, based on table 1, there were four patients excluded from the study as the surgery had been extended for more than three hours or they had required colloid or blood transfusion intraoperatively. The patients were relatively comparable in term of mean of age, body weight, duration of fasting and the duration of surgery. They did not differ in term of total volume of study solution and intraoperative blood loss as well.

Table 1. Patients demographic and perioperative variables.

\begin{tabular}{|c|c|c|c|}
\hline variables & Ringer's Lactate (n=71) (SD) & Balanced group $(n=70)(\mathrm{SD})$ & $P$-value* \\
\hline Age (years) & $5.58(3.04)$ & $5.73(2.71)$ & 0.756 \\
\hline Body weight (kg) & $19.71(10.65)$ & $17.81(7.90)$ & 0.235 \\
\hline Duration of fasting (hr) & $7.08(2.81)$ & $6.49(2.87)$ & 0.213 \\
\hline Duration of surgery (min) & $79.72(37.71)$ & $76.50(45.50)$ & 0.648 \\
\hline Volume of study solution (mls) & $319.45(244.16)$ & $263.57(171.27)$ & 0.118 \\
\hline Intraoperative blood loss (mls) & $51.55(40.24)$ & $46.14(61.53)$ & 0.537 \\
\hline
\end{tabular}

* independent t-test

Based on table 2, the comparison of means of pre-infusion values of electrolytes and acid base was made. Pre-infusion chloride level was significantly higher in sterofundin group patients, $(P=0.020)$ but was still in the normal range. In contrast, the lactate level in the Ringer's Lactate group patients was significantly above the normal value $(P=0.029)$ with the means of $1.73 \pm 0.69 \mathrm{mmol} / \mathrm{L}$. Other pre-infusion values are not statistically significant.

Table 2. Means of pre-infusion values of electrolytes and acid base.

\begin{tabular}{|c|c|c|c|}
\hline Parameters (mmol/L) & Ringer's Lactate (n= 71) (SD) & Sterofundin ${ }^{\circledR}$ ISO $(n=70)$ (SD) & P-value* \\
\hline \multicolumn{4}{|l|}{ Electrolytes } \\
\hline Sodium, $\mathrm{Na}+$ & $134.25(2.94)$ & $135.01(2.61)$ & 0.107 \\
\hline Chloride, Cl- & $107.31(3.20)$ & $109.33(6.49)$ & 0.020 \\
\hline Calcium, $\mathrm{Ca} 2+$ & $1.19(0.08)$ & $1.20(0.07)$ & 0.585 \\
\hline \multicolumn{4}{|l|}{ Acid base } \\
\hline $\mathrm{pH}$ & $7.33(0.07)$ & $7.34(0.62)$ & 0.368 \\
\hline Base Excess, BE- & $-2.50(2.81)$ & $-2.05(2.80)$ & 0.338 \\
\hline Bicarbonate, HCO3- & $21.97(2.27)$ & $22.04(3.04)$ & 0.863 \\
\hline Lactate & $1.73(0.69)$ & $1.49(0.58)$ & 0.029 \\
\hline Glucose & $5.36(0.84)$ & $5.27(0.66)$ & 0.479 \\
\hline
\end{tabular}

*Independent t-test

Table 3 showed significant difference of post-infusion means of serum Sodium, chloride and calcium concentration among two different groups. There was reduction of serum sodium level and increment of serum chloride and calcium in both type of fluid on post-infusion values.

Table 3. Means of post-infusion values of electrolytes and acid base.

\begin{tabular}{lllc}
\hline Parameters $(\mathbf{m m o l} / \mathbf{L})$ & Ringer's Lactate $(\mathbf{n}=\mathbf{7 1})(\mathbf{S D})$ & Sterofundin ${ }^{\circledR}$ ISO $(\mathbf{n}=\mathbf{7 0})(\mathbf{S D})$ & $\boldsymbol{P}$ - $\boldsymbol{v a l u}^{*}$ \\
\hline Electrolytes & & & \\
Sodium, $\mathrm{Na}+$ & $134.06(2.16)$ & $134.94(2.24)$ & 0.018 \\
Chloride, $\mathrm{Cl}-$ & $108.21(4.26)$ & $110.30(6.46)$ & 0.025 \\
Potassium, K+ & $4.19(0.46)$ & $4.12(0.49)$ & 0.358 \\
Calcium, Ca2+ & $1.19(0.08)$ & $1.22(0.08)$ & 0.014 \\
Acid base & & & \\
pH & $7.32(0.07)$ & $7.32(0.07)$ & 0.828 \\
pCO2 & $45.89(10.92)$ & $45.95(11.37)$ & 0.974 \\
Base Excess, BE- & $-2.46(2.60)$ & $-2.52(2.66)$ & 0.906 \\
Bicarbonate, HCO3- & $22.09(2.05)$ & $22.33(3.37)$ & 0.604 \\
Lactate & $1.76(0.77)$ & $1.30(0.54)$ & $<0.001$ \\
Glucose & $5.79(1.12)$ & $5.60(1.20)$ & 0.341 \\
\hline
\end{tabular}

*Independent t-test

Comparing table 4 and table 5, both treatment groups showed significant increase of serum chloride concentration 
over time i.e; from $107.31 \pm 3.20 \mathrm{mmol} / \mathrm{L}$ to $108.21 \pm 4.26$ $\mathrm{mmol} / \mathrm{L}(P=0.018)$ in Ringer's Lactate group and from $109.33 \pm 6.49 \mathrm{mmol} / \mathrm{L}$ to $110.30 \pm 6.56 \mathrm{mmol} / \mathrm{L}(P=0.027)$ in Sterofundin ${ }^{\circledR}$ ISO group. On the other hand, significant increase in calcium level was seen in sterofundin group with the mean of $1.20 \pm 0.07 \mathrm{mmol} / \mathrm{L}$ vs. $1.22 \pm 0.08 \mathrm{mmol} / \mathrm{L}$ $(P=0.002)$. There was also significant reduction and normalization in lactate level in sterofundin group $(P=0.017)$. Furthermore, there was significant acidosis $(P=0.022)$ and reduction of base excess level in sterofundin group when comparison of pre and post-infusion made. Both study solution resulted to significant increase of blood glucose level but no hyperglycemia encountered.

Table 4. Comparison of means between pre and post-infusion values of electrolytes and acid base in Ringer's Lactate group.

\begin{tabular}{|c|c|c|c|}
\hline Parameters (mmol/L) & Pre-infusion (n= 71) (SD) & Post-infusion (n=71) (SD) & $P$-value \\
\hline \multicolumn{4}{|l|}{ Electrolytes } \\
\hline Sodium, $\mathrm{Na}+$ & $134.25(2.94)$ & $134.06(2.16)$ & 0.416 \\
\hline Chloride, Cl- & $107.31(3.20)$ & $108.21(4.26)$ & 0.018 \\
\hline Potassium, $\mathrm{K}+$ & $4.05(0.44)$ & $4.19(0.46)$ & 0.009 \\
\hline Calcium, $\mathrm{Ca} 2+$ & $1.19(0.08)$ & $1.19(0.08)$ & 0.569 \\
\hline \multicolumn{4}{|l|}{ Acid base } \\
\hline $\mathrm{pH}$ & $7.33(0.07)$ & $7.32(0.07)$ & 0.371 \\
\hline $\mathrm{pCO} 2$ & $43.95(7.78)$ & $45.89(10.92)$ & 0.177 \\
\hline Base Excess, BE- & $-2.50(2.81)$ & $-2.46(2.60)$ & 0.883 \\
\hline Bicarbonate, $\mathrm{HCO} 3-$ & $21.97(2.27)$ & $22.09(2.05)$ & 0.614 \\
\hline Lactate & $1.73(0.69)$ & $1.76(0.77)$ & 0.704 \\
\hline Glucose & $5.36(0.84)$ & $5.79(1.12)$ & $<0.001$ \\
\hline
\end{tabular}

*Independent t-test

Table 5. Comparison of means between pre and post-infusion values of electrolytes and acid base in Sterofundin ${ }^{\circledR}$ ISO group.

\begin{tabular}{|c|c|c|c|}
\hline Parameters (mmol/L) & Pre-infusion $(n=70)($ SD) & Post-infusion (n=70) (SD) & P-value* \\
\hline \multicolumn{4}{|l|}{ Electrolytes } \\
\hline Sodium, $\mathrm{Na}+$ & $135.01(2.61)$ & $134.94(2.24)$ & 0.710 \\
\hline Chloride, $\mathrm{Cl}-$ & $109.33(6.49)$ & $110.30(6.56)$ & 0.027 \\
\hline Potassium, $\mathrm{K}+$ & $4.04(0.40)$ & $4.12(0.49)$ & 0.208 \\
\hline Calcium, $\mathrm{Ca} 2+$ & $1.20(0.07)$ & $1.22(0.08)$ & 0.002 \\
\hline \multicolumn{4}{|l|}{ Acid base } \\
\hline $\mathrm{pH}$ & $7.34(0.62)$ & $7.32(0.07)$ & 0.022 \\
\hline pCO2 & $22.04(3.04)$ & $22.33(3.37)$ & 0.554 \\
\hline Base Excess, BE- & $-2.05(2.80)$ & $-2.52(2.66)$ & 0.043 \\
\hline Bicarbonate, $\mathrm{HCO} 3-$ & $22.04(3.04)$ & $22.33(3.37)$ & 0.554 \\
\hline Lactate & $1.49(0.58)$ & $1.30(0.54)$ & 0.017 \\
\hline Glucose & $5.27(0.66)$ & $5.60(1.20)$ & 0.010 \\
\hline
\end{tabular}

*Independent t-test

Table 6 showed the mean differences between pre- and post-infusion values of electrolytes and acid base status between two different group. There only significant mean difference (pre and post-infusion values) between both solutions was in calcium level where sterofundin resulted to significant increase of calcium level $(P=0.015)$ with the mean difference of $0.020 \pm 0.06 \mathrm{mmol} / \mathrm{L}$ vs. $0.005 \pm 0.07 \mathrm{mmol} / \mathrm{L}$. The other parameters were found to be insignificant.

Table 6. Mean differences between pre-and post-infusion values of electrolytes and acid base status between two different group.

\begin{tabular}{lll}
\hline Parameters (mmol/L) & $\begin{array}{l}\text { Ringer's Lactate } \\
(\mathbf{n = 7 1 )}(\mathbf{S D})\end{array}$ & $\begin{array}{l}\text { Sterofundin }{ }^{\circledR} \text { ISO } \\
\text { (n=70) (SD) }\end{array}$ \\
\hline Electrolytes & & $\boldsymbol{P}$ - value* \\
Sodium, Na+ & $-0.20(2.03)$ & $-0.07(1.60)$ \\
Chloride, Cl- & $0.90(3.13)$ & $0.97(3.60)$ \\
Potassium, K+ & $0.14(0.45)$ & $0.08(0.52)$ \\
Calcium, Ca2+ & $0.005(0.07)$ & $0.020(0.06)$ \\
Acid base & & 0.902 \\
pH & $-0.001(0.08)$ & $-0.020(0.08)$ \\
pCO2 & $1.94(11.98)$ & $2.50(11.88)$ \\
Base Excess, BE- & $0.04(2.24)$ & $-0.47(1.89)$ \\
Bicarbonate, HCO3- & $0.12(2.04)$ & 0.435 \\
Lactate & $0.03(0.78)$ & 0.015 \\
Glucose & $0.43(1.05)$ & 0.356 \\
\hline
\end{tabular}

*Independent t-test 


\section{Discussion}

Vigilant fluid management is essential in perioperative period in the paediatric patients. Fluid therapies are designed to fulfill several requirements intraoperatively. These are, correcting the fluid deficit, intraoperative fluid maintenance and replacing the ongoing loss during surgery [1]. Adequate volume of fluid is needed to maintain adequate tissue perfusion and to counteract the effect of anaesthesia. The deficit might be contributed from preoperative fasting, intraoperative losses due to haemorrhage and losses from third space cavity. These third space losses which of extracellular fluid (ECF) might be significant as paediatric relatively has larger percentage of ECF as compared to the adult.

In the operating theatre, the fluid requirements may rapidly change during conduct of anaesthesia and surgery, alteration in body temperature and metabolism and fluid volume shift. Lindahl et al [21] found that the energy expenditure is reduced in anaesthetized children. Moreover, trauma, hemorrhage and tissue exposure is associated with shifting of body fluids between the compartments, necessitating fluid replacement to maintain electrolytes and acid base parameters [19]. Adequate plasma volume is important in maintain the cardiac output and hence tissue perfusion. Inadequate tissue perfusion is associated with poor outcome following surgery [22]. It is a matter of fact that, paediatric patients undergoing surgeries are subjected to wide ranges of intraoperative complications includes hemodynamic instability, acid-base and electrolytes imbalances. Hence, the most ideal type of intraoperative fluid is yet to be determined and remains debatable among clinicians.

There are vast selections of crystalloid solutions in the market. They differ in term of electrolytes contents and osmolarity. Generally, crystalloid with physiologically balanced electrolytes with osmolarity mimicking to human plasma is more preferred especially among paediatric populations. A physiological solution is a solution which has a balance electrolytes particularly, sodium, potassium, calcium, magnesium, chloride and glucose in concentrations physiologically proportionate to the plasma constituents [23]. Ringer's lactate and sterofundin ${ }^{\circledR}$ ISO are two types of commonly used crystalloids and contains almost balanced electrolytes. However, the former are slightly hypoosmolar to human plasma while the latter contains excessive level of chloride to maintain normal osmolarity. Infusion of such balanced solution devoid the risk of iatrogenic disruption but may potentially cause volume overload. This study was intended to find out any possible electrolyte changes between two groups of solution in minor surgical procedures involving paediatric patients with duration of surgery of less than 3 hours.

In our study, we found the significant changes of potinfusion plasma sodium and calcium on top of the chloride and lactate which differ since the pre-infusion period. As sterofundin contains higher concentration of sodium by 10 $\mathrm{mmol} / \mathrm{L}$, the end plasma sodium is better in sterofundin group. However, infusion of sterofundin does not leading to hypernatremia since it contains normal level of sodium. Apart from that, stimulation of ADH hormone secondary to stress response devoid the risk of hypernatremia and improve the intravascular volume. It is further supported with significant reduction in lactate level. However, monitoring of urine output and $\mathrm{ADH}$ level is required to determine the effect of ADH in maintaining plasma level of sodium. During perioperative period, $\mathrm{ADH}$ secretion is unpredictable and studies have shown deleterious effect of using the hypotonic solutions $[24,25]$. On the other hand, both fluids solutions contains excessive amount of chloride as compared to human plasma. Ringer's lactate contains $112 \mathrm{mmol} / \mathrm{L}$ and sterofundin contains $127 \mathrm{mmol} / \mathrm{L}$ of chloride ions. Thus, both fluids infusion lead to increase in serum chloride concentration at the end of the study. However, pre- and postinfusion comparison of the mean differences between both fluids is not significant. Suempelmann R et. al. [26] found that a minor alteration of electrolytes and acid base is harmless among paediatrics due to their larger extracellular space and buffering capacity of bicarbonate ions. In previous study, hyperchloremia was found in the patients infused with sterofundin concluded that the contents of chloride is excessive [27]. As expected, there was a significant improvement on mean difference of calcium level in the patients infused with sterofundin. It is matter of fact that sterofundin contains higher calcium level than Ringer's Lactate solution. In contrast, Disma et. al. [27] found significant reduction of the post infusion calcium level in younger paediatric patients underwent major surgery after infusion of sterofundin solution. There was also significant increase of the post infusion glucose level in both types of study solutions. Anaesthesia and stress response during surgery are responsible for this finding on top of the exogenous lactate which is utilized for gluconeogenesis [28]. However, acetate rarely produces increment of blood glucose level [29].

In term of acid base parameters, variable effects were seen in the patients infused with sterorofundin as intraoperative maintenance fluid. Reduction in post-infusion $\mathrm{pH}$ correlated with reduction in Base Excess level. However, there was also significant reduction in lactate level. This condition might be true since sterofundin has acetate and maleate as the buffer. In contrast, the acid base parameters are more stable in Ringer's Lactate group. Yoshikazu et. al. [29] found that acetated balanced solutions improved blood $\mathrm{pH}$, Base Excess and metabolic acidosis as compared to lactated solution. In this study, we speculated that short duration of surgery with lower blood and fluid losses and smaller volume of infusion of study solutions resulted to this finding. In addition, according to Wooten's multi-compartmental model, acid base chemistry is determined by both extracellular and intracellular effects including fluid redistribution between the compartments [30]. 


\section{Conclusion}

In conclusion, Sterofundin ${ }^{\circledR}$ ISO, a balanced crstalloid solution resulted to variable effect on electrolytes and acidbase balanced in paediatric surgical patients underwent minor surgery of less than 3 hours. This fluid was not found to be superior than Ringer's Lactate solution. However, in complex surgical procedures with major fluid shift, usage of balanced solution as intraoperative fluid might be beneficial and yet to be determined.

\section{Disclosures}

Ethics: This study was approved by "Ethical Board for Human Research, Universiti Sains Malaysia" (Jawatankuasa Etika Penyelidikan Manusia) USMKK/PPP/JEPeM/241.3 (8) on 10th October 2013.

\section{Funding}

The study was funded by departmental resources

\section{Conflict of Interest}

None declared.

\section{References}

[1] Holliday MA, Segar WE. The maintenance need for water in parenteral fluid therapy. Pediatrics. 1957; 19(5):823-32.

[2] Camboulives J. Fluid, transfusion, and blood sparing techniques. B Bissonnette BD Pediatric Anesthesia, principles \& practice New York: Mc Graw Hill. 2002:576-99.

[3] Paut O, Lacroix F. Recent developments in the perioperative fluid management for the paediatric patient. Current Opinion in anesthesiology. 2006; 19(3):268-77.

[4] Way C, Dhamrait R, Wade A, Walker I. Perioperative fluid therapy in children: a survey of current prescribing practice. British journal of anaesthesia. 2006; 97 (3):371-9.

[5] Murat I, DUBOIS MC. Perioperative fluid therapy in pediatrics. Pediatric anesthesia. 2008; 18(5):363-70.

[6] Holliday MA, Friedman AL, Segar WE, Chesney R, Finberg L. Acute hospital-induced hyponatremia in children: a physiologic approach. The Journal of pediatrics. 2004; 145 (5):584-7.

[7] Moritz ML, Ayus JC. Prevention of hospital-acquired hyponatremia: a case for using isotonic saline. Pediatrics. 2003; 111 (2):227-30.

[8] Zornow MH, Scheller MS, Shackford SR. Effect of a hypertonic lactated Ringer's solution on intracranial pressure and cerebral water content in a model of traumatic brain injury. Journal of Trauma and Acute Care Surgery. 1989; 29 (4):484-8.

[9] Holcroft JW, Trunkey DD. Extravascular lung water following hemorrhagic shock in the baboon: Comparison between resuscitation with Ringer's lactate and Plasmanate. Annals of surgery. 1974; 180(4):4.
[10] Kamp-Jensen M, Olesen K, Bach V, Schütten H, Engquist A. Changes in serum electrolyte and atrial natriuretic peptide concentrations, acid-base and haemodynamic status after rapid infusion of isotonic saline and Ringer lactate solution in healthy volunteers. British journal of anaesthesia. 1990; 64(5):606-10.

[11] Thomas D, Alberti K. Hyperglycaemic effects of Hartmann's solution during surgery in patients with maturity onset diabetes. British journal of anaesthesia. 1978; 50 (2):185-8.

[12] De Jonghe B, Cheval C, Misset B, Timsit J-F, Garrouste M, Montuclard L, et al. Relationship between blood lactate and early hepatic dysfunction in acute circulatory failure. Journal of critical care. 1999; 14(1):7-11.

[13] Guidet B, Soni N, Della Rocca G, Kozek S, Vallet B, Annane $\mathrm{D}$, et al. A balanced view of balanced solutions. Crit Care. 2010; 14(5):325.

[14] Gan T, Bennett-Guerrero E, Phillips-Bute B, Wakeling H, Moskowitz D, Olufolabi Y, et al. Hextend [registered sign], a Physiologically Balanced Plasma Expander for Large Volume Use in Major Surgery: A Randomized Phase III Clinical Trial. Anesthesia \& Analgesia. 1999;88(5):992-8.

[15] Wilkes NJ, Woolf R, Mutch M, Mallett SV, Peachey T, Stephens R, et al. The effects of balanced versus saline-based hetastarch and crystalloid solutions on acid-base and electrolyte status and gastric mucosal perfusion in elderly surgical patients. Anesthesia \& Analgesia. 2001; 93 (4):811-6.

[16] Wilkes NJ, Woolf RL, Powanda MC, Gan TJ, Machin SJ, Webb A, et al. Hydroxyethyl starch in balanced electrolyte solution (Hextend $\AA)$ - Pharmacokinetic and pharmacodynamic profiles in healthy volunteers. Anesthesia \& Analgesia. 2002; 94(3):538-44.

[17] Boldt J. Volume therapy in cardiac surgery: are Americans different from Europeans? Journal of cardiothoracic and vascular anesthesia. 2006; 20(1):98-1.

[18] Boldt J. II. The balanced concept of fluid resuscitation. British journal of anaesthesia. 2007; 99 (3):312-5.

[19] Nair SG, Balachandran R. Perioperative fluid and electrolyte management in paediatric patients. Indian $J$ Anaesth. 2004;48(5):355-64.

[20] Hadimioglu N, Saadawy I, Saglam T, Ertug Z, Dinckan A. The effect of different crystalloid solutions on acid-base balance and early kidney function after kidney transplantation. Anesthesia \& Analgesia. 2008; 107 (1):264-9.

[21] Lindahl S. Energy expenditure and fluid and electrolyte requirements in anesthetized infants and children. Anesthesiology. 1988; 69 (3):377-82

[22] Mythen MG, Hamilton MA. Hyperchloremic metabolic acidosis: is it clinically relevant? Transfusion alternatives in transfusion medicine. 2001; 3 (4):15-9.

[23] Dorje P, Adhikary G, Tempe DK. Avoiding iatrogenic hyperchloremic acidosis - call for a new crystalloid fluid. The Journal of the American Society of Anesthesiologists. 2000;92(2):625.

[24] Adelman R, Solhaug M. Pathophysiology of body fluids and fluid therapy. Nelson textbook of pediatrics Philadelphia, PA: WB Saunders. 2000:211-22. 
[25] Burrows FA, Shutack JG, Crone RK. Inappropriate secretion of antidiuretic hormone in a postsurgical pediatric population. Critical care medicine. 1983; 11 (7):527-31.

[26] Suempelmann R, Witt L, Bruett M, Osterkorn D, Koppert W, Osthaus WA. Changes in acid-base, electrolyte and hemoglobin concentrations during infusion of hydroxyethyl starch 130/0.42/6: 1 in normal saline or in balanced electrolyte solution in children. Pediatric Anesthesia. 2010; 20 (1):100-4.

[27] Disma N, Mameli L, Pistorio A, Davidson A, Barabino P, Locatelli BG, et al. A novel balanced isotonic sodium solution vs normal saline during major surgery in children up to 36 months: a multicenter RCT. Pediatric Anesthesia. 2014; 24 (9):980-6.
[28] Arai T, Inoue A, Uematsu Y, Sako T, Kimura N. Activities of enzymes in the malate-aspartate shuttle and the isoenzyme pattern of lactate dehydrogenase in plasma and peripheral leukocytes of lactating Holstein cows and riding horses. Research in veterinary science. 2003; 75 (1):15-9.

[29] Matsuda Y, Sakurai T, Iino M, Nakayama K. Comparative study on the effects of acetated Ringer's solution, lactated Ringer's solution, Ringer's solution, and 5\% glucose-acetated Ringer's solution on canine hemorrhagic shock. Journal of anesthesia. 1994;8(3):326-33

[30] Wooten EW. Calculation of physiological acid-base parameters in multicompartment systems with application to human blood. Journal of Applied Physiology. 2003; 95 (6):2333-44. 